\title{
BMJ Open Analysis on geographic variations in hospital deaths and endovascular therapy in ischaemic stroke patients: an observational cross-sectional study in China
}

\author{
Hui Chen, ${ }^{1,2}$ Lizheng Shi, ${ }^{3}$ Ni Wang, ${ }^{1,2}$ Yangtong Han, ${ }^{4}$ Yilu Lin, ${ }^{3}$ Mingfeng Dai, ${ }^{5}$ \\ Honglei Liu, ${ }^{1,2}$ Xiao Dong, ${ }^{6}$ Ming Xue, ${ }^{5}$ Hua Xu ${ }^{6}$
}

To cite: Chen H, Shi L, Wang N, et al. Analysis on geographic variations in hospital deaths and endovascular therapy in ischaemic stroke patients: an observational cross-sectional study in China. BMJ Open 2019;9:e029079. doi:10.1136/ bmjopen-2019-029079

- Prepublication history and additional material for this paper are available online. To view please visit the journal (http:// dx.doi.org/10.1136/bmjopen2019-029079).

$\mathrm{HC}$ and LS contributed equally.

Received 11 January 2019 Revised 28 May 2019 Accepted 29 May 2019
Check for updates

\section{(C) Author(s) (or their} employer(s)) 2019. Re-use permitted under CC BY-NC. No commercial re-use. See rights and permissions. Published by BMJ.

For numbered affiliations see end of article.

Correspondence to

Dr Hua Xu; hua.xu@uth.tmc.edu

\section{ABSTRACT}

Objectives Stroke is the leading cause of death and adult disability in China, following a rise in incidence over the last few decades. We aimed to explore the geographic variations in hospital mortality and endovascular therapy (EVT) use among ischaemic stroke (IS) patients in China, and investigate the associated potential risk factors.

Design Observational cross-sectional study of patients hospitalised for stroke.

Setting Hospital discharge data for 1267 tertiary hospitals between 1 January 2015 and 31 December 2015 were derived from the Nationwide Hospital Discharge Database operated by the National Health Commission of China.

Participants 1826332 patients aged $\geq 18$ years, hospitalised following stroke.

Outcome measures In-hospital mortality and EVT use. Results The nationwide hospital mortality rate of IS patients was $0.88 \%$ (95\% Cl $0.86 \%$ to $0.90 \%)$; there was a significantly greater risk of mortality in the Northeast (OR 2.37; 95\% Cl 2.23 to 2.52), West (1.65; 1.54 to 1.78$)$, South $(1.25 ; 1.17$ to 1.33$)$ and North $(1.29 ; 1.20$ to 1.39$)$ than in the East. Tertiary B hospitals (OR 1.05; 95\% Cl 1.00 to 1.09), patients admitted from emergency departments and older patients were associated with higher hospital mortality. The national EVT use rate was $0.45 \%(95 \% \mathrm{Cl} 0.44 \%$ to $0.46 \%$ ). Compared with in East China, EVT use was significantly lower in the Northeast $(0 \mathrm{R} 0.22 ; 95 \% \mathrm{Cl}$ 0.20 to 0.24$)$ and West $(0.64 ; 0.58$ to 0.71$)$, though not the North (1.23; 1.14 to 1.33). Tertiary A hospitals (OR 2.62; $95 \% \mathrm{Cl} 2.43$ to 2.83 ), male patients and patients admitted from emergency departments were also associated with higher EVT use rates.

Conclusions There were substantial disparities in mortality and EVT use for hospitalised patients with IS among China's tertiary hospitals, linked with both geographic and hospital characteristics. More targeted intervention at regional and hospital levels is needed for providing effective health technologies and eventually improving post-stroke outcomes.

\section{Strengths and limitations of this study}

- This is the first study to investigate regional variations in stroke outcomes using real-world hospital discharge data from 1276 tertiary hospitals in China, involving $>1.8$ million inpatients diagnosed with stroke.

- The detailed analyses provided important insight into the geographic and hospital variations in stroke outcomes, which may be helpful in promoting the appropriate use of endovascular therapy (EVT) and reducing unnecessary variations in treatment for ischaemic stroke (IS) patients.

- The analysed data were derived from an administrative database which lacks clinical information, restricting the clinical analyses of potential risk factors for hospital mortality rates and EVT use for IS patients.

\section{INTRODUCTION}

Stroke is the second leading cause of mortality and the second most common cause of long-term serious disability globally. ${ }^{12}$ It was also the leading cause of death and adult disability in China, where 1.8 million people died from stroke in 2016, accounting for nearly one-third of the global deaths from stroke. ${ }^{34}$ China was estimated to have the highest incidence of stroke worldwide, with $>12.4$ million stroke survivors $\geq 40$ years of age in 2016. ${ }^{35}$ The stroke burden in China is excessively increased in line with most other countries of the world, ${ }^{6}$ and continues to increase due to the ageing of the population and an increasing prevalence trend of risk factors. ${ }^{7}$

Stroke prognosis varies substantially among patients, and has many potential causes, including diversity in sex, race, age, stroke subtype, comorbidity and treatment 
strategy. ${ }^{8-11}$ Over the last decade, developments in endovascular therapy (EVT) (such as intra-arterial thrombolysis and mechanical thrombectomy) have brought about promising new prospects for treating acute ischaemic stroke (IS), ${ }^{12}$ especially when intravenous thrombolysis is contraindicated or has failed. The frequency of EVT use has increased substantially in China, but varies by region, such as between rural and urban areas, partly because of inconsistent medical development and economic levels. ${ }^{13}$ However, previous studies have used data from specific regions ${ }^{10}$ or selected hospitals. ${ }^{14}$ Therefore, identifying the determinants of hospital mortality rates and EVT use among IS patients is likely an important step in developing targeted interventions to improve access to EVT and reduce mortality rates. Accordingly, a nationwide investigation using representative samples is needed.

Since 2013, the National Health Commission (NHC) of China has collected hospital discharge data. In the present cross-sectional study, hospital discharge data from 2015 were used to investigate how geographic and hospital characteristics are linked to hospital mortality and EVT use for IS inpatients in tertiary hospitals in China.

\section{METHODS}

\section{Data source}

The study data used herein were derived from the Nationwide Hospital Discharge Database (NHDD) ${ }^{15}$ operated by the Center for Health Statistics and Information, the NHC of China. The centre had audited the data, which were also double-checked and deidentified before any further authorised use. The NHDD was accessed remotely with permission and data was analysed in an anonymous manner.

Summarised information about hospitalised patients is collected routinely from all secondary hospitals (with numbers of beds between 100 and 500) and tertiary hospitals (with over 500 beds), except for traditional Chinese medicine hospitals, national hospitals, army hospitals and hospitals located in Tibet. This information is imported into the NHDD every 3 months via a private network interface. The information includes patients' demographics, medical insurance status, source of hospital admissions, length of hospital stay, discharge deposition and diagnoses and procedures.

\section{Study design and population}

For very few secondary hospitals are able to perform EVT, only data from tertiary hospitals were used in the present study. We exported data from all 1854443 hospitalisations discharged from 1276 tertiary hospitals between 1 January 2015 and 31 December 2015, with a primary diagnosis of stroke (identified using the International Classification of Diseases 10th Revision (ICD-10) codes I60-63, I66 and I67.8) ${ }^{16}$ To restrict our study to a typical adult stroke population, we excluded 8426 hospitalisations involving those aged $<18$ years. We excluded 3027 records with missing survival status, and 16658 with length of stay of 0 day (as they may not reflect a stroke diagnosis) or $\geq 180$ days. The final study sample therefore included 1,826,332 distinct hospitalisation episodes.

\section{Outcomes}

The primary outcome of interest was hospital mortality, defined as the in-hospital case-fatality rate for patients hospitalised with stroke. Death information was obtained from the discharge deposition field. We also calculated the admissions per 10000 resident population in each of the geographic regions using the total resident population of that region reported in the China Statistical Yearbook 2015. ${ }^{17}$ The secondary outcome was the use of EVT, including intra-arterial thrombolysis, mechanical thrombectomy, stent implantation and balloon angioplasty, identified by the International Classification of Diseases Clinical Modification 9th Revision codes 99.10, $39.74,00.63-65$ and $00.61-62$ in any of the primary and secondary procedural fields. ${ }^{18} 19$

\section{Patient and hospital characteristics}

Patient characteristics included sex, age (grouped into $18-59,60-69,70-79$ and $\geq 80$ years $^{20}$, taking the sample balance into consideration), source of hospital admission (emergency department, clinic and referral) and diagnostic correctness (identified by the agreement between diagnosis at admission and primary diagnosis at discharge). Medical insurance status (urban employee basic medical insurance (UEBMI) for urban employees and retirees, urban residents basic medical insurance for urban non-employees, new rural cooperative medical scheme for rural population and others) was also included as a potential patient factor, which may impact the choice of hospital and the affordability of a certain treatment. Stroke was categorised into three main subtypes: subarachnoid haemorrhage (SAH), identified by ICD-10 code I60, intracerebral haemorrhage (ICH) by I61 and IS by I63. ${ }^{6}$ We also calculated the Charlson Comorbidity Index $(\mathrm{CCI})^{21}$ for each patient, using the adaptation to predict in-hospital or short-term mortality. ${ }^{22}{ }^{23}$ This index is based on the presence or absence of 17 different kinds of conditions during the primary hospitalisation, which includes disorders such as renal disease, liver disease, pulmonary disease, diabetes mellitus, peripheral arterial disease and cancer. Clinical conditions within the CCI system were identified using ICD-10 codes in any secondary diagnosis field. ${ }^{24}$ The higher the CCI score, the heavier the severity of the comorbidity. A CCI score of zero indicated the absence of any Charlson comorbidity.

The hospital characteristic included in this study was hospital level. Tertiary hospitals are accredited into levels A (higher level) and B (lower level) by national health authorities according to their service, management, technology, equipment and so on. This information was obtained from hospitals' official websites. We also identified the hospital's location. There are six administrative regions in mainland China: North, Northeast, East, South, Southwest and Northwest. The study populations 
were very small in the Southwest and Northwest; therefore, those two regions were merged into 'West'. The entire country was thus divided into five geographic regions in this study.

\section{Statistical analysis}

Statistical analyses used IBM SPSS Statistics V.23.0. Patient and hospital characteristics were compared among geographic regions using one-way analysis of variance for scale variables, Pearson's $\chi^{2}$ test for nominal variables and the Kruskal-Wallis test for ordinal variables. Hospital mortality rate was also adjusted for age because of the considerable differences in age among the five geographical regions, where the age constituent in the North was used as the reference. $\mathrm{P}<0.05$ was considered statistically significant.

More detailed analyses were conducted on IS patients in this study because that the vast majority of the stroke patients were ischaemic, and the most important that there were available and accessible therapies to benefit these patients. To explore associations between geographic regions and hospital mortality, we used multivariable logistic regression analysis, controlling for patient characteristics (age, sex, medical insurance status, source of hospital admission, diagnostic correctness and CCI score) and hospital characteristic (hospital level). We also performed similar analysis to examine the associations between geographic regions and EVT use. Adjusted ORs with 95\% CIs were used to report the results of multivariable logistic regression analyses.

\section{Patient and public involvement}

Patients and the general public were not involved in this study.

\section{RESULTS}

\section{Overview of the study population}

We identified a total of 1826332 hospital admissions for adult patients with a primary diagnosis of stroke from 1276 tertiary hospitals. Among them, 27.2\% and 26.6\% were from hospitals located in the South and Northeast, respectively, while less than half of the above patients $(12.3 \%$ and $12.6 \%$, respectively) were from hospitals in the West and North. Of note, admissions per 10000 resident population in the Northeast were the highest among the five geographic regions, which was almost fivefold those in the West (44.2 vs 7.6; figure 1).

The nationwide proportions were $79.1 \%, 15.5 \%$ and $3.4 \%$ for patients with IS, ICH and SAH, respectively. There were also statistical differences in the constituent ratio of stroke subtype among geographic regions $(p<0.001$; figure 2A). There were a total of 30631 $(1.68 \%)$ deaths during the hospitalisation stay. The crude nationwide hospital mortality rates of SAH, ICH and IS were $4.34 \%, 5.15 \%$ and $0.88 \%$, respectively. These varied significantly among geographic regions, with the highest rates in the Northeast $(8.11 \%, 9.04 \%$ and $1.24 \%)$ and the lowest in the East $(2.19 \%, 2.73 \%$ and $0.56 \%$ ) (all p values $<0.001$; figure 2B).

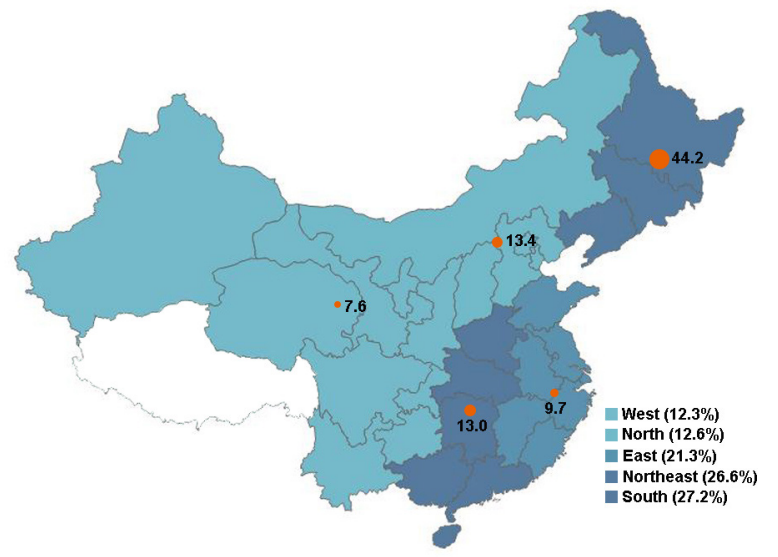

Figure 1 Hospital admissions of stroke in the five geographic regions. Numbers displayed inside the map were admissions per 10000 resident population in each region, and the sizes of the orange dots are proportional to the number. The figure was drawn by using the Tableau V.10.4 software with its built-in map of China.

\section{Basic characteristics of IS patients}

We identified a total of 1443894 hospital admissions for adult patients with a primary IS diagnosis. A predominance of male inpatients was seen in all five regions. The age constitution varied significantly among regions $(p<0.001)$; patients in the North were the youngest on
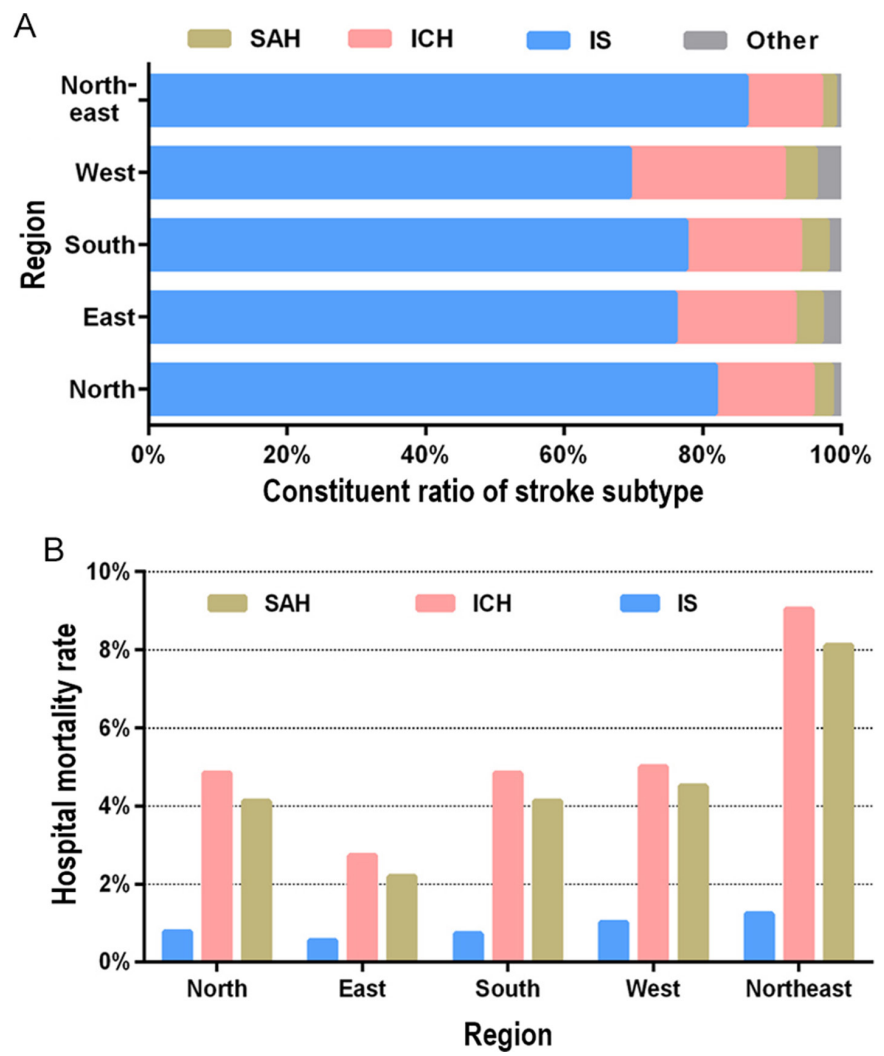

Figure 2 Characteristics of stroke inpatients in the five geographical regions. (A) Constituent ratio of stroke subtype. (B) Crude hospital mortality of patients with subarachnoid haemorrhage $(\mathrm{SAH})$, intracerebral haemorrhage $(\mathrm{ICH})$ and ischaemic stroke (IS). 


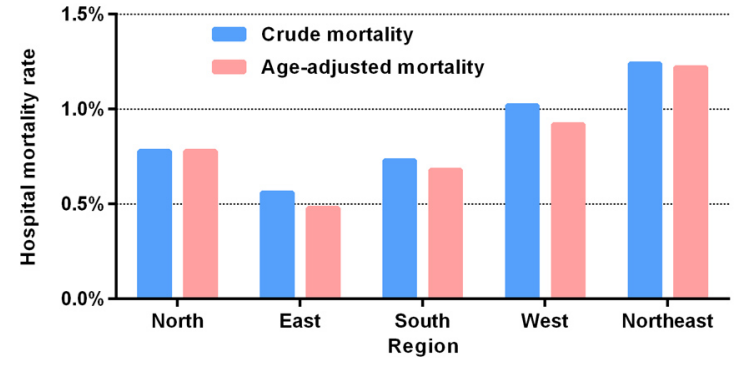

Figure 3 Regional variations in crude and adjusted hospital mortality following ischaemic stroke. Hospital mortality rate was adjusted for age with the age constituent in the North as a reference.

average. Those in the North and South were more likely to have severe comorbidities than those in other regions. There was significant variation in the proportion of admissions from tertiary level A hospitals (range: 64.9\%$72.6 \%)$ across geographic regions $(\mathrm{p}<0.001$; online supplementary table $\mathrm{S} 1$ ).

\section{Hospital mortality of IS patients}

The nationwide mortality rate of IS patients was $0.88 \%$ (95\% CI $0.86 \%$ to $0.90 \%$ ), ranging from $0.56 \%$ in the East to $1.24 \%$ in the Northeast. After being adjusted for age, they still varied significantly among regions $(p<0.001)$, ranging from $0.48 \%$ in the East to $1.22 \%$ in the Northeast (figure 3).

After controlling for other potential factors, we found a strong association between the geographic region and hospital mortality risk of the IS patients with the lowest risk in the East. Inpatient mortality was also linked to the hospital level, with a higher level indicating a lower mortality risk. Patients admitted from the emergency department had a higher mortality risk than those admitted from the clinic. The incorrect diagnosis at admission suggested a higher death risk in hospitals than the opposite. Other risk factors were older age, severer comorbidity and having UEBMI as the main medical insurance (table 1).

\section{EVT use for IS patients}

A total of 6480 EVTs were performed. The nationwide EVT use rate was $0.45 \%$ (95\% CI $0.44 \%$ to $0.46 \%$ ), and differed significantly among geographic regions, with the lowest of $0.13 \%$ in the Northeast and the highest of $0.81 \%$ in the North. Among four main kinds of EVT, stent implantation was most popular, where the use rate $(0.30 \%)$ was even higher than the sum of those for the other three kinds of EVT (figure 4A). A considerable number of IS patients received two or more kinds of EVT, and the most popular EVT combination was intra-arterial thrombolysis and stent implantation. More than three-quarters of IS patients in the East underwent stent implantation (alone or combined), which was almost twice the proportion in the Northeast (figure 4B).
Hospital level was an associated factor for EVT use; that is, more patients with IS admitted to hospitals of a higher level intended to receive EVT. Older patients tended not to receive EVT except for patients aged 60-69 years, whereas male patients with IS were more likely to receive EVT than females. Other potential factors contributing to a more frequent use of EVT included admission from the emergency department, moderate comorbidities and having UEBMI as the main medical insurance (table 2).

\section{DISCUSSION}

In this large-scale, cross-sectional study, the NHDD was used to investigate hospital mortality for stroke patients as well as EVT use for IS inpatients discharged from 1276 tertiary hospitals in China in 2015. The NHDD is quite similar to the Nationwide Inpatient Sample (NIS) database in terms of structure, contents and coverage. The NIS database was sponsored by the US Healthcare Cost and Utilization Project, and has been widely used in $>3600$ observational studies on hospitalisation characteristics. ${ }^{25}$ However, very few studies have used NHDD data. ${ }^{15}$ We first drew on this data source for a large observational study on stroke in China. This study investigated variations in hospital mortality in stroke patients among five geographic regions, and offered an in-depth analysis on the potential risk factors of hospital mortality and EVT use for IS. The results of this national study based on routinely collected discharge data can provide a better understanding of these variations in order to promote an appropriate use of EVT and further improve the outcome for IS patients.

In this study population of over 1.8 million inpatients with stroke, $>1.44$ million $(79.1 \%)$ were with IS. This aligned with another study on Chinese population in which IS accounted for $77.8 \%$ among prevalent stroke, ${ }^{26}$ and another study on population from some developed countries, wherein up to $67.3 \%-80.5 \%$ of stroke cases were attributed to IS. ${ }^{27}$ This proportion of IS patients varied substantially among geographic regions, with the highest $(86.2 \%)$ in the Northeast. The national hospital mortality rate of stroke was $1.68 \%$, wherein patients with ICH showed higher mortality than those with SAH and IS. This is partly due to ICH patients were usually accompanied with cerebral oedema and intracranial hypertension and some severer complications such as cardiovascular disease, stress ulcer and lung infection than IS patients. ${ }^{28}{ }^{29}$ We further found substantial differences in hospital mortality rates of stroke inpatients among regions. Of note, patients in the Northeast consistently had the highest mortalities in terms of the overall stroke and each subtype of stroke (ie, ICH, SAH and IS).

Our detailed analyses conducted on data for IS patients showed regional differences in hospital mortality and EVT use were substantial. Several factors may contribute to this. First, differences in hospital mortality may be 
Table 1 Factors associated with hospital mortality for patients with ischaemic stroke

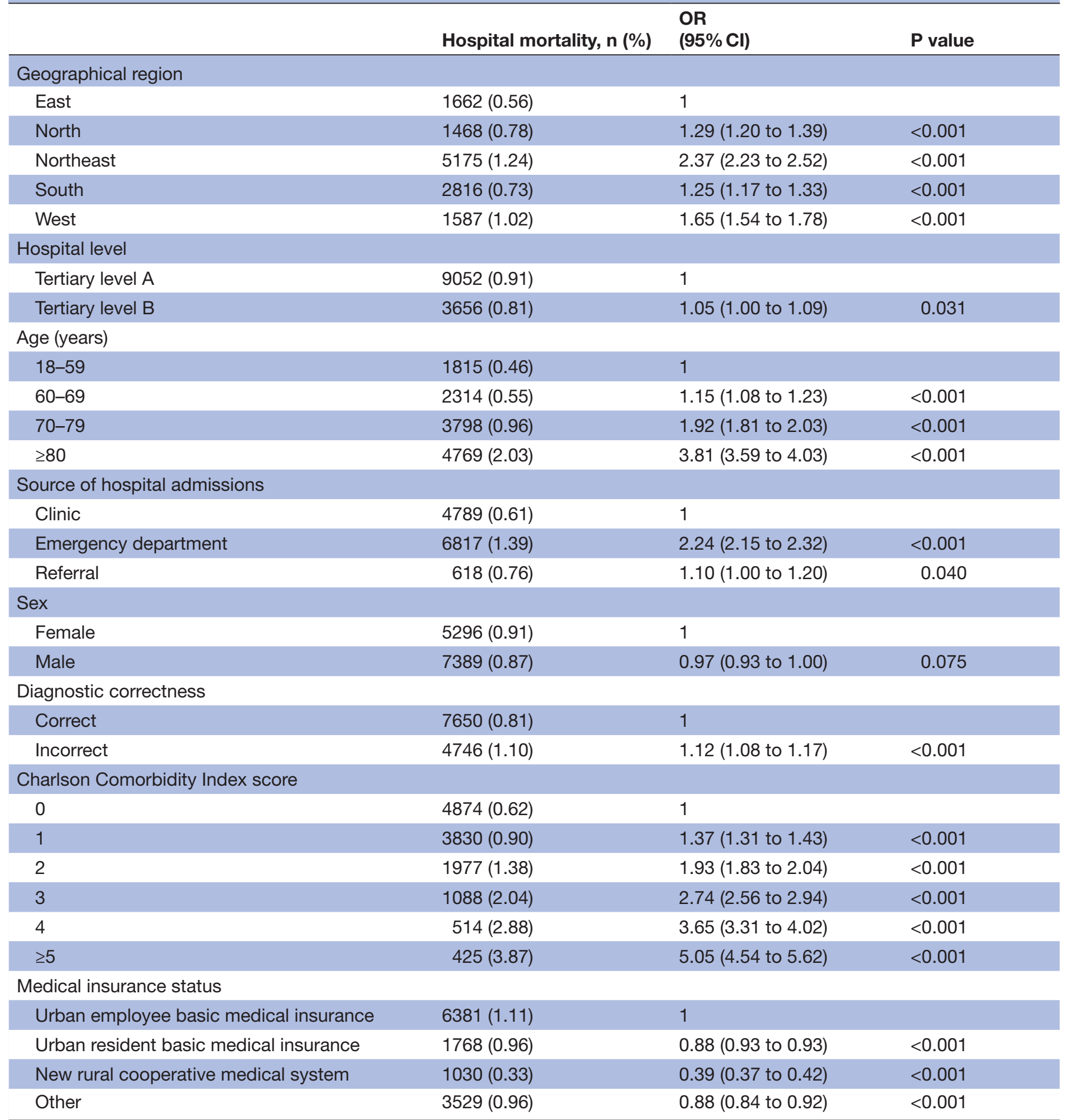

mainly due to the differences in lifestyle and inconsistent medical development and economic level among the regions. It was reported that the patients from northern China (including the Northeast and North regions in this study) tended to consume more alcohol and smoke more cigarettes, and were more likely to have family histories of stroke, cerebral ischaemia and heart disease, as well as higher body mass index. ${ }^{30}$ This is one of the potential explanation for why both the crude and age-adjusted hospital mortalities in the Northeast were the substantially highest among the five regions. Additionally, differences in the use of preventative therapy, control of vascular risk factors and the advances in acute stroke care among geographic regions may also contribute to the variation of hospital mortality. ${ }^{31}$

In China, tertiary hospitals are usually comprehensive or general hospitals at the city, province or national level, providing specialised medical and health services to the 

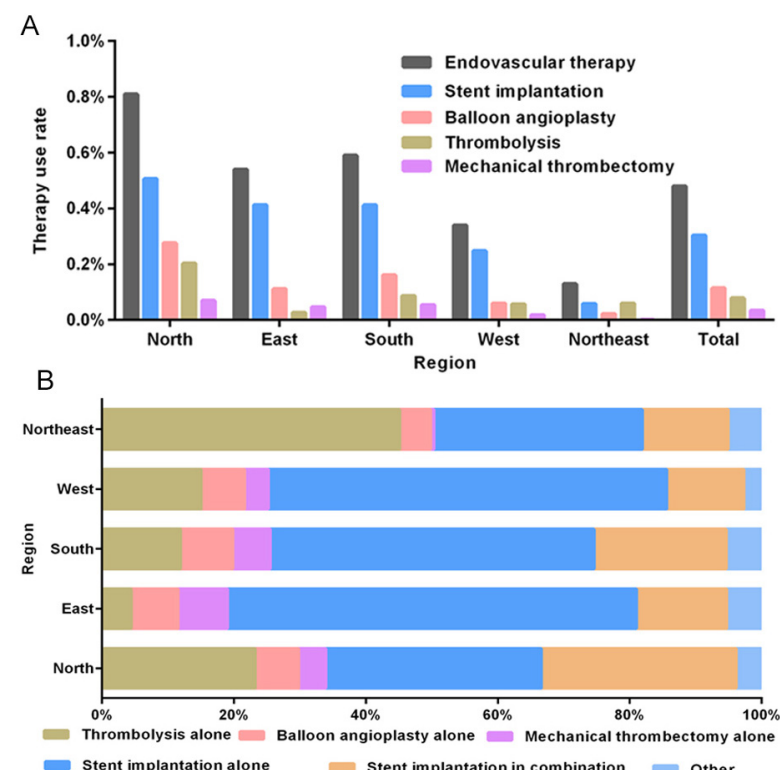

Figure 4 Endovascular therapy in the five geographical regions. (A) Rates of four different therapies used. (B) Constituent ratio of different therapies.

surrounding areas. Based on the institute size and medical management, technology and quality, they are further subdivided into levels A and B. ${ }^{27}$ Tertiary level A hospitals may be larger and more organised, and have more resources and support necessary for providing an early recognition, accurate diagnosis, targeted therapy and a continuous high level of care to stroke patients. ${ }^{13}$ There were increasing evidences that high-volume and highlevel hospitals tend to have relatively higher success rates for EVT, ${ }^{32}$ which may improve survival for IS patients. Therefore, it was not surprising to find that level A hospitals were generally associated with a higher rate of performing EVT, along with a lower mortality for IS patients.

Apart from the hospital level, correct diagnosis at admission, and therefore timely treatment provided by experienced doctors, were also important towards the survival of stroke patients. Patients with IS presenting to the emergency departments over 3 hours after onset of symptoms reportedly had a higher incidence of hospital mortality than those presenting within 3 hours. ${ }^{5}$ Our findings also suggested that incorrect diagnosis of patients at admission led to a higher mortality risk because of the possible delay in providing effective treatment. In our study population, there were substantial geographic differences in both proportions of patients admitted at a tertiary level A hospital and those who were admitted with a correct diagnosis. These may contribute to the regional disparities in overall hospital mortality rates. Moreover, IS patients presenting to emergency departments possibly have much more severe complications than those presenting to clinics, leading to an increased mortality. ${ }^{34}{ }^{35}$ However, once diagnosed with IS, the patient was more likely to be offered EVT. ${ }^{32}$ This may further introduce geographic differences in hospital death rate and EVT use, because the proportion of patients admitted from the emergency department also varied considerably among geographic regions in this study.

Age is another potentially important reason for the regional variations in hospital mortality rate and EVT use. Our study, like others, ${ }^{11}{ }^{32} 36$ reported higher stroke hospital mortality and lower EVT use in older patients, especially in patients at age of $\geq 80$ years who were not eligible for thrombolysis treatment. ${ }^{37}$ This was probably due to severer comorbidities and more difficult catheterisation of older patients, ${ }^{8}$ along with relatively higher rate of death and disability associated with EVT. The higher proportion of older patients in some regions may therefore be connected to the higher overall hospital mortality rates and lower overall frequency in EVT use in those regions. Differences in patient characteristics, such as the severity of common comorbidities and medical insurance status, are other potential reasons for regional variations in hospital mortality rate and EVT use.

Although our study showed a higher rate of death was usually accompanied by less frequent use of EVT among IS patients, especially in the Northeast and West, it remains unclear whether the outcomes of IS patients treated with EVT are better than those who undergo no treatment. This is because other changes in practice, such as better patient selection, more technical expertise and better periprocedural care, may also explain some improvement in clinical outcomes. ${ }^{38-40}$

\section{Limitations}

A major limitation of this study is that the analysed data did not consist of clinical data, which restricted the clinical analyses of potential risk factors for mortality rates and EVT use for stroke patients. Some information about the exact time, such as when patients arrived at the hospital and had the first medical contact were also unavailable. These factors are important for doctors to choose the most appropriate treatment for IS patients. Additionally, some severely ill patients may have stopped treatment and been discharged alive from hospitals to die at home, or because they could not afford to remain in the hospital. The survival status for these patients was uncertain, and could result in an underestimation of mortality.

\section{CONCLUSION}

More than 1.8 million inpatients diagnosed with stroke were investigated in this large, nationwide study of hospitalised patients. We observed considerable disparities in hospital mortality and EVT use for IS patients admitted to tertiary hospitals across China. These disparities were linked to the geographic locations and hospital characteristic. More work at the provincial, regional and national levels is needed to develop 
Table 2 Factors associated with EVT use for ischaemic stroke patients

\begin{tabular}{|c|c|c|c|}
\hline & EVT use, n (\%) & OR $(95 \% \mathrm{Cl})$ & $P$ value \\
\hline \multicolumn{4}{|l|}{ Region } \\
\hline East & $1605(0.54)$ & 1 & \\
\hline North & $1523(0.81)$ & $1.23(1.14$ to 1.33$)$ & $<0.001$ \\
\hline Northeast & $548(0.13)$ & $0.22(0.20$ to 0.24$)$ & $<0.001$ \\
\hline South & $2276(0.59)$ & 1.02 (0.95 to 1.09$)$ & 0.635 \\
\hline West & $528(0.34)$ & 0.64 (0.58 to 0.71$)$ & $<0.001$ \\
\hline \multicolumn{4}{|l|}{ Hospital level } \\
\hline Tertiary level B & $900(0.20)$ & 1 & \\
\hline Tertiary level A & $5580(0.56)$ & 2.62 (2.43 to 2.83 ) & $<0.001$ \\
\hline \multicolumn{4}{|l|}{ Age, years } \\
\hline $18-59$ & $2259(0.58)$ & 1 & \\
\hline $60-69$ & $2447(0.58)$ & 1.04 (0.98 to 1.10$)$ & 0.197 \\
\hline $70-79$ & $1554(0.39)$ & 0.73 (0.68 to 0.78$)$ & $<0.001$ \\
\hline$\geq 80$ & $218(0.09)$ & 0.17 (0.14 to 0.19$)$ & $<0.001$ \\
\hline \multicolumn{4}{|l|}{ Source of hospital admission } \\
\hline Clinic & $3143(0.40)$ & 1 & \\
\hline Emergency department & $2872(0.58)$ & 1.54 (1.46 to 1.62$)$ & $<0.001$ \\
\hline Referral & $194(0.24)$ & 0.91 (0.78 to 1.06$)$ & 0.214 \\
\hline \multicolumn{4}{|l|}{ Sex } \\
\hline Female & $1512(0.26)$ & 1 & \\
\hline Male & $4966(0.58)$ & 2.00 (1.89 to 2.13$)$ & $<0.001$ \\
\hline \multicolumn{4}{|l|}{ Charlson Comorbidity Index score } \\
\hline 0 & $3241(0.41)$ & 1 & \\
\hline 1 & $2176(0.51)$ & $1.17(1.10$ to 1.23$)$ & $<0.001$ \\
\hline 2 & $711(0.50)$ & 1.15 (1.06 to 1.26$)$ & 0.001 \\
\hline 3 & $230(0.43)$ & 0.97 (0.84 to 1.11$)$ & 0.631 \\
\hline 4 & $80(0.45)$ & $1.00(0.79$ to 1.27$)$ & 0.999 \\
\hline$\geq 5$ & $42(0.38)$ & 0.79 (0.56 to 1.10$)$ & 0.156 \\
\hline \multicolumn{4}{|l|}{ Medical insurance status } \\
\hline Urban employee basic medical insurance & $2526(0.44)$ & 1 & \\
\hline Urban resident basic medical insurance & $786(0.43)$ & 1.01 (0.93 to 1.10$)$ & 0.863 \\
\hline New rural cooperative medical system & $1333(0.42)$ & 0.87 (0.81 to 0.93$)$ & $<0.001$ \\
\hline Other & $1835(0.50)$ & $1.17(1.10$ to 1.24$)$ & $<0.001$ \\
\hline
\end{tabular}

EVT, endovascular therapy.

targeted interventions for reducing disparities and unnecessary variations, improving access to effective health technologies, enhancing the overall quality of stroke treatment and care and ultimately improving stroke outcomes.

\section{Author affiliations}

${ }^{1}$ School of Biomedical Engineering, Capital Medical University, Beijing, China ${ }^{2}$ Beijing Key Laboratory of Fundamental Research on Biomechanics in Clinical Application, Capital Medical University, Beijing, China

${ }^{3}$ Department of Global Health Management and Policy, Tulane University, New Orleans, Louisiana, USA

${ }^{4}$ Department of Neurology, Ji Shui Tan Hospital and Fourth Medical College of Peking University, Beijing, China
${ }^{5}$ Center for Health Statistics and Information, National Health and Family Planning Commission of the Peoples Republic of China, Beijing, China

${ }^{6}$ School of Biomedical Informatics, University of Texas Health Science Center at Houston, Houston, Texas, USA

Contributors $\mathrm{HX}$ and LS designed the study and developed the methods. MX and MD collected data. HC, NW and YL sorted and analysed the data. $\mathrm{HC}, \mathrm{XD}$ and $\mathrm{YH}$ drafted the manuscript. HL and NW prepared the figures and tables. LS and HX provided critical review of the manuscript. All authors have reviewed and approved the final version of the manuscript for publication.

Funding This work was supported by the National Natural Science Foundation of China (No 81671786 and No 81701792).

Map disclaimer The depiction of boundaries on the map(s) in this article do not imply the expression of any opinion whatsoever on the part of BMJ (or any member 
of its group) concerning the legal status of any country, territory, jurisdiction or area or of its authorities. The map(s) are provided without any warranty of any kind, either express or implied.

Competing interests None declared.

Patient consent for publication Not required.

Provenance and peer review Not commissioned; externally peer reviewed.

Open access This is an open access article distributed in accordance with the Creative Commons Attribution Non Commercial (CC BY-NC 4.0) license, which permits others to distribute, remix, adapt, build upon this work non-commercially, and license their derivative works on different terms, provided the original work is properly cited, appropriate credit is given, any changes made indicated, and the use is non-commercial. See: http://creativecommons.org/licenses/by-nc/4.0/.

\section{REFERENCES}

1. GBD 2016 Causes of Death Collaborators. Global, regional, and national age-sex specific mortality for 264 causes of death, 19802016: a systematic analysis for the Global Burden of Disease Study 2016. Lancet 2017;390:1151-210.

2. GBD 2016 DALYs and HALE Collaborators. Global, regional, and national disability-adjusted life-years (DALYs) for 333 diseases and injuries and healthy life expectancy (HALE) for 195 countries and territories, 1990-2016: a systematic analysis for the Global Burden of Disease Study 2016. Lancet 2017;390:1260-344.

3. GBD 2016 Stroke Collaborators. Global, regional, and national burden of stroke, 1980-2016: a systematic analysis for the Global Burden of Disease Study 2016. Lancet Neurol 2019.

4. Feigin VL, Nguyen G, Cercy K, et al. Global, Regional, and CountrySpecific Lifetime Risks of Stroke, 1990 and 2016. N Engl J Med 2018;379:2429-37.

5. Wang L, Liu J, Yang Y, et al. Essentials of report on the prevention for Chinese stroke 2017. Chinese Journal of Cerebrovascular Diseases 2018;15:611-7.

6. Feigin VL, Norrving B, George MG, et al. Prevention of stroke: a strategic global imperative. Nat Rev Neurol 2016;12:501-12.

7. Wu S, Wu B, Liu M, et al. Stroke in China: advances and challenges in epidemiology, prevention, and management. Lancet Neurol 2019;18:394-405.

8. Fischer U, Arnold M, Nedeltchev K, et al. Impact of comorbidity on ischemic stroke outcome. Acta Neurol Scand 2006;113:108-13.

9. Ayala C, Croft JB, Greenlund KJ, et al. Sex differences in US mortality rates for stroke and stroke subtypes by race/ethnicity and age, 1995-1998. Stroke 2002;33:1197-201.

10. Fu X, Wong KS, Wei JW, et al. Factors associated with severity on admission and in-hospital mortality after primary intracerebral hemorrhage in China. Int J Stroke 2013;8:73-9.

11. Willey JZ, Ortega-Gutierrez S, Petersen N, et al. Impact of acute ischemic stroke treatment in patients $>80$ years of age: the specialized program of translational research in acute stroke (SPOTRIAS) consortium experience. Stroke 2012;43:2369-75.

12. Mokin M, Dumont TM, Veznedaroglu E, et al. Solitaire flow restoration thrombectomy for acute ischemic stroke: retrospective multicenter analysis of early postmarket experience after FDA approval. Neurosurgery 2013;73:19-26.

13. Adamczyk P, Attenello F, Wen G, et al. Mechanical thrombectomy in acute stroke: utilization variances and impact of procedural volume on inpatient mortality. J Stroke Cerebrovasc Dis 2013;22:1263-9.

14. Yang X, Li Z, Zhao X, et al. Use of warfarin at discharge among acute ischemic stroke patients with nonvalvular atrial fibrillation in China. Stroke 2016;47:464-70.

15. Chen H, Shi L, Xue M, et al. Geographic Variations in In-Hospital Mortality and Use of Percutaneous Coronary Intervention Following Acute Myocardial Infarction in China: A Nationwide Cross-Sectional Analysis. J Am Heart Assoc 2018;7:e008131.

16. Benjamin EJ, Blaha MJ, Chiuve SE, et al. Heart disease and stroke statistics-2017 update: a report from the American Heart Association. Circulation 2017;135:e146-e603.

17. National Bureau of Statistics of China. China Statistical Yearbook 2015. Beijing: China Statistics Publishing House, 2015.
18. Hassan AE, Chaudhry SA, Grigoryan M, et al. National trends in utilization and outcomes of endovascular treatment of acute ischemic stroke patients in the mechanical thrombectomy era. Stroke 2012;43:3012-7.

19. Beijing hospital management institute. $C N-D R G$ s grouping scheme. Beijing: China Medical Science and Technology Press, 2015.

20. Ohinmaa A, Zheng Y, Jeerakathil T, et al. Trends and Regional Variation in Hospital Mortality, Length of Stay and Cost in Hospital of Ischemic Stroke Patients in Alberta Accompanying the Provincial Reorganization of Stroke Care. J Stroke Cerebrovasc Dis 2016;25:2844-50.

21. Charlson ME, Pompei P, Ales KL, et al. A new method of classifying prognostic comorbidity in longitudinal studies: development and validation. J Chronic Dis 1987;40:373-83.

22. Unsal A, Resorlu B, Atmaca AF, et al. Prediction of morbidity and mortality after percutaneous nephrolithotomy by using the Charlson Comorbidity Index. Urology 2012;79:55-60.

23. Falsetti L, Viticchi G, Tarquinio N, et al. Charlson comorbidity index as a predictor of in-hospital death in acute ischemic stroke among very old patients: a single-cohort perspective study. Neurol Sci 2016;37:1443-8.

24. Sundararajan V, Henderson T, Perry C, et al. New ICD-10 version of the Charlson comorbidity index predicted in-hospital mortality. J Clin Epidemiol 2004;57:1288-94.

25. Agency for Healthcare Research and Quality, Rockville, MD. HCUP Reports. Healthcare Cost and Utilization Project (HCUP). 2019 http:// www.hcup-us.ahrq.gov/reports.jsp (Accessed 6 Apr 2019).

26. Wang $\mathrm{W}$, Jiang $\mathrm{B}$, Sun $\mathrm{H}$, et al. Prevalence, incidence, and mortality of stroke in China: results from a nationwide population-based survey of 480687 adults. Circulation 2017;135:759-71.

27. Feigin VL, Lawes CM, Bennett DA, et al. Stroke epidemiology: a review of population-based studies of incidence, prevalence, and case-fatality in the late 20th century. Lancet Neurol 2003;2:43-53.

28. Hemphill JC, Greenberg SM, Anderson CS, et al. Guidelines for the management of spontaneous intracerebral hemorrhage: a guideline for healthcare professionals from the American Heart Association/ American Stroke Association. Stroke 2015;46:2032-60.

29. Chinese-Stroke-Society. Guidelines for diagnosis and treatment of intracerebral hemorrhage in China. Chinese Journal of Neurology 2015;48:435-44.

30. Pu Y, Liu L, Wang Y, et al. Geographic and sex difference in the distribution of intracranial atherosclerosis in China. Stroke 2013;44:2109-14.

31. Sun H, Zou X, Liu L. Epidemiological factors of stroke: a survey of the current status in china. J Stroke 2013;15:109-14.

32. Menon BK, Saver JL, Goyal M, et al. Trends in endovascular therapy and clinical outcomes within the nationwide Get With The Guidelines-Stroke registry. Stroke 2015;46:989-95.

33. Gupta R, Horev A, Nguyen T, et al. Higher volume endovascular stroke centers have faster times to treatment, higher reperfusion rates and higher rates of good clinical outcomes. J Neurointerv Surg 2013;5:294-7.

34. Wira CR, Rivers E, Martinez-Capolino C, et al. Cardiac complications in acute ischemic stroke. West J Emerg Med 2011;12:414-20.

35. Akhtar N, Kamran S, Singh R, et al. Prolonged stay of stroke patients in the emergency department may lead to an increased risk of complications, poor recovery, and increased mortality. J Stroke Cerebrovasc Dis 2016;25:672-8.

36. Yang $Q$, Tong X, Schieb L, et al. Vital signs: recent trends in stroke death rates - United States, 2000-2015. MMWR Morb Mortal Wkly Rep 2017;66:933-9.

37. Chinese-Stroke-Society. Guidelines for diagnosis and treatment of acute ischemic stroke in China. Chinese Journal of Neurology 2015;48:246-57.

38. Campbell BC, Mitchell PJ, Kleinig TJ, et al. Endovascular therapy for ischemic stroke with perfusion-imaging selection. N Engl J Med 2015;372:1009-18

39. Singer OC, Haring HP, Trenkler J, et al. Periprocedural aspects in mechanical recanalization for acute stroke: data from the ENDOSTROKE registry. Neuroradiology 2013;55:1143-51.

40. Broderick JP, Palesch YY, Demchuk AM, et al. Endovascular therapy after intravenous t-PA versus t-PA alone for stroke. N Engl J Med 2013;368:893-903. 